\title{
Uso da Microalbuminúria em Amostras Urinárias Aleatórias para 0 Rastreamento de Pré-eclâmpsia em Grávidas Diabéticas
}

\author{
Use of Microalbuminuria in Random Urine Samples to Screen \\ Diabetic Pregnant Women for Preeclampsia \\ Victor Hugo Saucedo Sanchez, Anna Maria Bertini, Wladimir Correa Taborda, \\ Rosy Ane Araujo Barros, Cristiane Cadore Hagemann
}

\begin{abstract}
RESUMO
Objetivo: avaliar o desempenho da dosagem de microalbuminúria como método para rastreamento de pré-eclampsia

Métodos: estudo prospectivo longitudinal no qual foram incluidas 45 grávidas diabéticas. Foi quantificada a microalbuminúria em três periodos distintos da gravidez: antes da $18^{a}$ semana, entre a $18^{a}$ e a $24^{a}$ semana e entre a $32^{a}$ e a $36^{a}$ semana de gravidez. Todas as pacientes freqüentaram o pré-natal entre janeiro de 2000 e dezembro de 2001. O ensaio de microalbuminúria/creatinina é método quantitativo para medir baixas concentrações de albumina, creatinina e a relação albumina/creatinina na urina. Como critério indicativo de dano renal incipiente e risco para pré-eclâmpsia foi empregada a relação albumina/creatinina maior que $16 \mathrm{mg} / \mathrm{g}$. A sensibilidade, especificidade, valor preditivo positivo e valor preditivo negativo da relação albumina/creatinina foram calculados para predizer a ocorrência ou ausência de pré-eclâmpsia.

Resultados: do total de pacientes, $17 \%$ apresentaram pré-eclâmpsia. A sensibilidade da relação albumina/creatinina foi crescente de 12,5\% com 18 semanas para 25\% entre 18 e 24 semanas e 87\% após a 32 ${ }^{a}$ semana. Em contraste, a especificidade teve valor decrescente de 97 para 89 e $83 \%$, respectivamente. O valor preditivo positivo foi relativamente baixo, com valores de 50, 33 e 53\% nos três diferentes periodos de avaliação. De outro modo, o valor preditivo negativo foi elevado nas três faixas de idade gestacional, com valores de 83, 84 e 96\%.

Conclusões: a quantificação aleatória da microalbuminúria pôde predizer corretamente a não ocorrência de pré-eclâmpsia em grávidas diabéticas, sendo pouco eficiente para a identificação correta das pacientes que evoluíram com pré-eclâmpsia.
\end{abstract}

PALAVRAS-CHAVE: Microalbuminúria. Diabete melito. Pré-eclâmpsia. Complicações da gravidez.

\section{Introdução}

A nefropatia diabética é complicação crônica do diabete associada a elevada taxa de mortalidade, sendo uma das principais causas de insuficiência renal crônica em adultos. Está também

Departamento de Obstetrícia da Universidade Federal de São Paulo

Correspondência:

Victor Hugo Saucedo Sanchez

Av. Sargento Geraldo Santana, 1100 , bloco 4, apto 31

04674-000 - São Paulo - SP

Telefone : (11) 5524-8198

e-mail: vhs-sanchez@uol.com.br associada a elevada morbidade perinatal, particularmente devida ao aumento das taxas de prematuridade, especialmente em decorrência do desenvolvimento de pré-eclampsia, que ocorre em $35 \%$ a $55 \%$ das grávidas diabéticas nefropatas ${ }^{1}$.

A microalbuminúria significa a excreção urinária subclínica e anormal de albumina, não detectável por métodos bioquímicos usuais. Definida como aumento anormal da excreção urinária de albumina superior a $30 \mathrm{mg} / 24 \mathrm{~h}$ e inferior a $300 \mathrm{mg} / 24 \mathrm{~h}$, apresenta fisiopatologia multifatorial que determina maior passagem transcapilar glomerular de albumina, decorrente de lesões estruturais glomerulares ou altera- 
ções tubulares, podendo ser influenciada também por fatores genéticos e raciais ${ }^{2}$. A taxa de excreção de albumina é elevada em grávidas normais no terceiro trimestre de gravidez em relação aos dois primeiros trimestres, e, também, em comparação ao estado não gravídico ${ }^{3}$. Desta forma, a detecção de microalbuminúria durante o primeiro trimestre pode indicar dano renal incipiente em pacientes com elevado risco para complicações hipertensivas. Microalbuminúria persistente indica alta probabilidade de dano à filtração glomerular, sendo de relevância para diagnóstico precoce de nefropatia diabética ${ }^{4}$. A prevalência de microalbuminúria nos diabéticos não insulinodependentes varia entre 8 e 43\%. Sua presença se associa a obesidade, hipertensão arterial e dislipidemia, por mecanismo patogênico comum: a resistência à insulina ${ }^{5}$. Durante a gravidez, poderia atuar, ainda, como teste capaz de predizer o desenvolvimento de pré-eclampsia ${ }^{6}$. Diversos estudos realizados com grávidas diabéticas nefropatas entre 1981 e 1994 demonstraram que a pré-eclâmpsia complicou mais de um terço das gestações $^{7,8}$. Da mesma forma, dois estudos demonstraram que a pré-eclâmpsia pode ser tão comum em pacientes com nefropatia incipiente quanto naquelas com nefropatia plenamente instalada. Winocour e Taylor ${ }^{9}$ relataram que três de quatro pacientes com nefropatia incipiente desenvolveram pré-eclâmpsia. Combs et al. ${ }^{10}$ observaram que a taxa de pré-eclâmpsia em grávidas diabéticas depende do nível de proteinúria no início da gravidez. A doença hipertensiva ocorreu em $10 \%$ das 204 pacientes sem nefropatia que apresentavam microalbuminúria no início da gravidez. Houve desenvolvimento de pré-eclâmpsia em 40\% das 45 grávidas que apresentavam nefropatia incipiente e proteinúria entre $190 \mathrm{mg} /$ dia e 499 $\mathrm{mg} /$ dia em $47 \%$ entre as 62 grávidas nefropatas que revelaram proteinúria superior a $500 \mathrm{mg} /$ dia no início da gravidez. Esta relação entre proteinúria precoce e pré-eclâmpsia persiste mesmo após análise de regressão múltipla e correção para fatores como a paridade, presença de hipertensão crônica ou retinopatia e controle glicêmico. Giordano et al. ${ }^{11}$ avaliaram 89 pacientes entre a $24^{\mathrm{a}}$ e a $32^{\mathrm{a}}$ semana de gravidez, dosando a microalbuminúria e a relação cálcio/creatinina para detecção precoce de pré-eclâmpsia. Observaram que a microalbuminúria apresentou sensibilidade de $16,3 \%$, especificidade de $95 \%$, valor preditivo positivo (VPP) de 25\% e negativo (VPN) de 83,8\%. Concluíram que a dosagem de microalbuminúria constitui teste diagnóstico com maior acurácia do que a relação cálcio/creatinina, embora as diferenças não tenham sido tão amplas para que se considere este o método de escolha para o rastreamento da pré-eclâmpsia em função de seu baixo
VPP. Resultados similares foram reportados por Baker e Hackett ${ }^{12}$.

O objetivo do presente estudo é avaliar o desempenho da dosagem de microalbuminúria como método para rastreamento de pré-eclâmpsia em grávidas diabéticas.

\section{Pacientes e Métodos}

O desenho de estudo aprovado pelo Comitê de Ética e Pesquisa da UNIFESP, foi do tipo prospectivo longitudinal (coorte) para validação da relação albumina/creatinina como teste de rastreamento para a ocorrência de pré-eclâmpsia em grávidas diabéticas. As pacientes foram submetidas à quantificação da proteinúria de 24 horas na primeira consulta, de modo a excluir grávidas diabéticas com função renal anormal, definida por proteinúria superior a $300 \mathrm{mg} / 24 \mathrm{~h}$, assim como grávidas com urocultura positiva antes da $18^{\mathrm{a}}$ semana de gravidez. Foram incluídos 45 grávidas diabéticas que freqüentaram o pré-natal no Centro de Diabetes da Universidade Federal de São Paulo (UNIFESP) entre janeiro de 2000 e dezembro de 2001.

O diagnóstico de diabete prévio à gravidez foi estabelecido pela constatação do uso de insulina e/ou hipoglicemiantes orais na primeira consulta de pré-natal. O diagnóstico de diabete melito foi precedido, em muitos casos, por rastreamento rotineiro realizado durante a assistência pré-natal ${ }^{13}$. O diagnóstico de diabete gestacional foi definido como intolerância a carboidratos em graus variáveis de gravidade, com inicio ou primeiro reconhecimento na gravidez ${ }^{14}$. Para o diagnóstico de pré-eclâmpsia consideramos os critérios do "National High Blood Pressure Education Program Working Group on High Blood Pressure in Pregnancy"15.

A incidência de pré-eclâmpsia foi de 17\% (oito pacientes) e a prevalência de hipertensão arterial crônica foi de 15\% (sete grávidas). As 33 pacientes restantes evoluíram somente com diabetes melito, sem complicações hipertensivas. A idade materna variou entre 19 e 42 anos, com média de 30 anos. Quanto à classificação do diabete, vinte e duas pacientes (49\%) apresentaram diabete do tipo II, em doze casos $(27 \%)$ o diagnóstico foi de diabete gestacional, sendo as onze pacientes remanescentes (24\%) classificadas como diabete tipo I. Todas as pacientes foram acompanhadas por equipe multidisciplinar visando o controle rigoroso da doença materna. A meta durante o pré-natal foi manter os niveis glicêmicos em valores inferiores a $100 \mathrm{mg} / \mathrm{dL}$ durante todo o dia, às custas de dieta e/ou utilização de insulina em regimes variados. As consultas foram agendadas em intervalos 
variáveis de acordo com a gravidade de cada caso, mas nunca superiores a três semanas.

Para este estudo, foram obtidas amostras de urina em três momentos distintos durante a evolução da gravidez: a primeira antes da $18^{a}$ semana de idade gestacional, a segunda entre a $18^{\mathrm{a}}$ e a $24^{\mathrm{a}}$ semana e a terceira entre a $32^{\mathrm{a}}$ e a $36^{\mathrm{a}}$ semana. A coleta de urina foi realizada de forma aleatória pela manhã. O valor de $16 \mathrm{mg} / \mathrm{g}$ foi estabelecido como limite superior da normalidade da relação albumina/ creatinina. A dosagem de microalbuminúria foi quantitativa por meio do aparelho analisador DCA 2000 (Bayer), adequado para medir baixas concentrações de albumina, creatinina e da relação albumina/creatinina na urina. O método foi desenvolvido para uso em laboratórios descentralizados, utilizando amostras de uma noite ou de tempo determinado e para quando se precisa dos resultados de forma imediata, já que o mesmo está disponivel em sete minutos.

O desempenho da microalbuminúria como teste de rastreamento para pré-eclâmpsia em grávidas diabéticas foi avaliado pela comparação dos resultados na presença ou ausência da moléstia no período anteparto. Calcularam-se a sensibilidade, a especificidade e os VPP e VPN de cada teste.

\section{Resultados}

A Tabela 1 apresenta o desempenho da relação albumina/creatinina como um teste de rastreamento de pré-eclâmpsia quando realizada antes da $18^{\mathrm{a}}$ semana de gravidez em 45 grávidas diabéticas. Demonstrou-se que a relação foi positiva (superior a $16 \mathrm{mg} / \mathrm{g}$ ) em dois casos, sendo que destes, somente um evoluiu com pré-eclâmpsia, determinando falso-positivo de 50\% (VPP de 50\%). Entre os 43 casos em que o teste foi negativo (inferior a $16 \mathrm{mg} / \mathrm{g}$ ) observou-se que 37 pacientes de fato não apresentaram a doença, configurando falso-negativo de $17 \%$ (VPN de $83 \%$ ).

Tabela 1 - Relação albumina/creatinina antes da $18^{\text {a }}$ semana de gravidez e ocorrência de pré-eclâmpsia em 45 grávidas diabéticas.

\begin{tabular}{ccccc}
\hline & & \multicolumn{3}{c}{ Pré-eclâmpsia } \\
& & $\begin{array}{c}\text { Presente } \\
\text { n }\end{array}$ & $\begin{array}{c}\text { Ausente } \\
\text { n }\end{array}$ & $\begin{array}{c}\text { Total } \\
\text { n }\end{array}$ \\
\hline Relação & Positiva & 1 & 1 & 2 \\
albumina/creatinina & Negativa & 7 & 36 & 43 \\
& Total & 8 & 37 & 45
\end{tabular}

Sensibilidade: $12,5 \%$

Especificidade: $97,0 \%$

Valor preditivo positivo: $50,0 \%$

Valor preditivo negativo: $83,0 \%$

Acurácia: $82,0 \%$
A Tabela 2 apresenta o desempenho da relação albumina/creatinina realizada entre a $18^{\mathrm{a}}$ e a $24^{a}$ semana de gravidez. A relação foi positiva (superior a $16 \mathrm{mg} / \mathrm{g}$ ) em 6 casos, sendo que destes, dois evoluíram com pré-eclâmpsia, determinando falso-positivo de 67\% (VPP de 33\%). Entre os 39 casos em que o teste foi negativo (inferior a $16 \mathrm{mg} / \mathrm{g}$ ) observou-se que 33 pacientes não manifestaram a doença, configurando falso-negativo de $16 \%$ (VPN de $83 \%$ ).

Tabela 2 - Relação albumina/creatinina entre a $18^{\mathrm{a}}$ e a $24^{\mathrm{a}}$ semana de gravidez e ocorrência de pré-eclâmpsia em 45 grávidas diabéticas.

\begin{tabular}{ccccc}
\hline & & \multicolumn{3}{c}{ Pré-eclâmpsia } \\
& & Presente & Ausente & Total \\
& & $\mathbf{n}$ & $\mathbf{n}$ & $\mathbf{n}$ \\
\hline Relação & Positiva & 2 & 4 & 6 \\
albumina/creatinina & Negativa & 6 & 33 & 39 \\
& Total & 8 & 37 & 45 \\
\hline
\end{tabular}

Sensibilidade: $25,0 \%$

Especificidade: $89,0 \%$

Valor preditivo positivo: $33,0 \%$

Valor preditivo negativo: $84,0 \%$

Acurácia: $77,0 \%$

A Tabela 3 apresenta o desempenho da relação albumina/creatinina como teste de rastreamento de pré-eclâmpsia quando realizado entre a $32^{\mathrm{a}}$ e a $36^{\mathrm{a}}$ semana de gravidez, em 44 pacientes, devido a parto prematuro de uma paciente. Observamos que a relação foi positiva (superior a $16 \mathrm{mg} / \mathrm{g}$ ) em treze casos, sendo que destes, sete evoluíram com pré-eclâmpsia, com falso-positivo de 47\% (VPP de 53\%). Entre os 31 casos em que o teste foi negativo (inferior a 16 $\mathrm{mg} / \mathrm{g})$ verificamos que 30 pacientes não manifestaram a doença, configurando falsonegativo de 4\% (VPN de 96\%).

Tabela 3 - Relação albumina/creatinina entre a $32^{\mathrm{a}}$ e a $36^{\mathrm{a}}$ semana de gravidez e ocorrência de pré-eclâmpsia em 44 grávidas diabéticas.

\begin{tabular}{ccccc}
\hline & & \multicolumn{3}{c}{ Pré-eclâmpsia } \\
& & $\begin{array}{c}\text { Presente } \\
\mathbf{n}\end{array}$ & $\begin{array}{c}\text { Ausente } \\
\mathbf{n}\end{array}$ & $\begin{array}{c}\text { Total } \\
\mathbf{n}\end{array}$ \\
\hline Relação & Positiva & 7 & 6 & 13 \\
albumina/creatinina & Negativa & 1 & 30 & 31 \\
& Total & 8 & 36 & 44 \\
\hline
\end{tabular}

Sensibilidade: $87,0 \%$

Especificidade: $83,0 \%$

Valor preditivo positivo: $53,0 \%$

Valor preditivo negativo: $96,0 \%$

Acurácia: $84,0 \%$

Ao analisarmos a evolução da sensibilidade da relação albumina/creatinina ao longo da gravidez, verificamos sensibilidade crescente em 
direção ao termo, atingindo 87\% quando o teste foi realizado entre 32 e 36 semanas de gravidez. Em contraste, a evolução da especificidade da relação albumina/creatinina foi decrescente ao longo da gravidez, variando entre $97 \%$ (antes da $18^{\mathrm{a}}$ semana) e $83 \%$ (entre a $32^{\mathrm{a}}$ e a $36^{\mathrm{a}}$ semana). O valor preditivo positivo da relação albumina/ creatinina variou entre $33 \%$ (entre a $18^{\mathrm{a}}$ e a $24^{\mathrm{a}}$ semana) e $53 \%$ (além da $32^{a}$ semana). Os resultados falso-negativos foram decrescentes com a evolução da gravidez, variando entre 17\% (antes da $18^{\mathrm{a}}$ semana) e 4\% (após a $32^{\mathrm{a}}$ semana).

\section{Discussão}

Existe consenso de que os distúrbios hipertensivos complicam a gravidez diabética mais freqüentemente do que o fazem nas gestações normais, fato geralmente atribuído à vasculopatia diabética subjacente. Outra possibilidade a ser considerada é que a hiperinsulinemia materna, como a que pode ser observada em grávidas resistentes à insulina portadoras de diabete do tipo II ou em pacientes diabéticas do tipo I com niveis elevados de insulina exógena, parece estar geralmente associada à hipertensão arterial sistêmica ${ }^{16}$.

Assim, o rastreamento de pré-eclâmpsia em grávidas diabéticas representa importante desafio para o obstetra, que deve tentar antecipar a ocorrência da doença e minimizar os seus efeitos deletérios para grávida e concepto. Verificamos a ocorrência de sindromes hipertensivas em 12 casos do nosso grupo de estudo (26,6\%), sendo que a pré-eclâmpsia complicou $17 \%$ das grávidas. Este resultado foi similar ao de Cousins ${ }^{17}$, que, em revisão de 24 séries publicadas entre 1965 e 1985 incluindo 791 pacientes, apurou que 15\% das gestações diabéticas evoluíram com distúrbios hipertensivos. Da mesma forma, Sandoval et al. ${ }^{18}$ acompanharam 186 grávidas com diabete melito durante o período de janeiro de 1992 a dezembro de 1993 e observaram que 17\% apresentaram préeclâmpsia, principal morbidade materna assinalada. Giordano et al. ${ }^{11}$ também apontam incidência de $18 \%$ de pré-eclâmpsia em tais grávidas.

O presente estudo avaliou a eficácia da dosagem de microalbuminúria como teste de rastreamento para pré-eclâmpsia em grávidas diabéticas, pela determinação da relação albumina/ creatinina. O kit de avaliação da microalbuminúria é muito simples e de fácil aplicabilidade, já que utiliza amostras aleatórias de urina, sendo o jejum desnecessário. Além disso, apresenta como vantagem a rapidez do resultado, disponivel em cerca de sete minutos. Mesmo com tais características, o teste pareceu-nos pouco apropriado para o rastreamento de pré-eclâmpsia em grávidas di- abéticas em função da alta porcentagem de resultados falso-positivos (47 a 67\%) e da baixa sensibilidade verificada nas diferentes faixas de idade gestacional, mesmo em população de alto risco para a pré-eclâmpsia. Cumpre assinalar que o período no qual o teste poderia ser aplicado situase entre a $18^{\mathrm{a}}$ e a $24^{\mathrm{a}}$ semana de gravidez. Nesta fase o processo fisiopatológico está completamente instalado, embora a doença ainda curse fase pré-clínica, caracterizando momento importante para a prevenção secundária. Nossos resultados na aplicação do teste de microalbuminúria entre a $18^{\mathrm{a}}$ e a $24^{\mathrm{a}}$ semana ficaram aquém da expectativa, com 67\% de falso-positivos (VPP de 33\%) e $16 \%$ de falso-negativos (VPN de 84\%). De maneira similar, Schröder et al. ${ }^{19}$ demonstraram que a detecção da microalbuminúria em grávidas diabéticas para predizer a manifestação de préeclâmpsia apresentou alto VPN (98\%) e baixo VPP (46\%), com sensibilidade de $85 \%$ e especificidade de $92 \%$. Neste estudo, em que a incidência de préeclâmpsia foi de $6,9 \%$, os autores consideram o teste útil no manejo de gestações diabéticas.

A porcentagem de falso-positivos foi relevante mesmo em idades gestacionais superiores $(32$ a 36 semanas). Verificamos que a microalbuminúria foi positiva em treze casos, sendo que destes, sete evoluíram com pré-eclâmpsia, determinando falso-positivo de 47\% (VPP de 53\%). Entre os 31 casos em que o teste foi negativo (inferior a $16 \mathrm{mg} / \mathrm{g}$ ) observou-se que 30 pacientes não manifestaram a doença, configurando falso-negativo de 4\% (VPN de 96\%). Estes resultados indicam que o teste apresenta aplicabilidade clinica limitada em função de a doença em questão geralmente apresentar sintomas clínicos evidentes nesta faixa de idade gestacional. Do mesmo modo, a dosagem da microalbuminúria antes da $18^{\mathrm{a}}$ semana apresentou resultados pouco promissores para rastrear corretamente a pré-eclâmpsia na fase pré-clinica, já que verificamos taxa de falso-positivo de 50\% (VPP de 50\%) e de falso-negativo de 17\% (VPN de 83\%).

Os resultados também demonstraram haver progressão crescente da sensibilidade ao longo da gravidez e redução progressiva da especificidade na mesma medida. Assim, a sensibilidade evoluiu de $12,5 \%$ antes da $18^{\mathrm{a}}$ semana para $87 \%$ próximo ao termo. Fica fácil entender, portanto, que a ocorrência de proteinúria, micro ou macro, seja mais comum a partir de 32 semanas, momento em que a pré-eclâmpsia está clinicamente manifesta, gerando mais resultados positivos do que nas faixas de idade gestacional mais precoces. De forma coerente, a especificidade variou em razão inversa à idade gestacional, ou seja, foi maior no início e menor no final da gravidez. Da mesma forma que Nakamura et $a .{ }^{20}$, consideramos que o teste apresenta aplicabilidade clínica, já que demons- 
trou-se capaz de identificar corretamente as grávidas que não apresentaram a doença em até $96 \%$, embora o resultado positivo seja pouco eficiente para a identificação correta das pacientes que evoluiram com pré-eclâmpsia.

\section{ABSTRACT}

Purpose: the aim of the present study was to evaluate the accuracy of microalbuminuria to predict preeclampsia. Methods: a prospective study of 45 consecutive diabetic gestations that were tested for microalbuminuria before the 18 th week, between the $18^{\text {th }}$ and $24^{\text {th }}$ week and between the 32 nd and 36th week of gestation. All patients had their prenatal care done from January 2000 to December 2001. The DCA 2000 microalbumin/creatinine assay is a quantitative method for measuring low concentrations of albumin, creatinine and the albumin/creatinine ratio in urine. According to laboratory standards, an albumin/creatinine ratio $>16 \mathrm{mg} / \mathrm{g}(1.8 \mathrm{mg} / \mathrm{mmol})$ indicates incipient renal damage and risk for preeclampsia. The sensitivity, specificity, positive and negative predictive values of the albumin/creatinine ratio were determined to predict the occurrence or the absence of preeclampsia, diagnosed through clinical criteria.

Results: of all patients, $17 \%$ developed preeclampsia. The sensitivity of albumin/creatinine ratio increased from $12.5 \%$ at 18 weeks to $25 \%$ between the 18 th and 24 th week and to $87 \%$ after the 32nd week. On the other hand, specificity presented a decreasing value from 97 to 89 and 83\%, respectively). The positive predictive value was relatively low in the three different periods of evaluation (50, 33 and $53 \%$, respectively. The negative predictive value was increased in the three stages of gestational age (83, 84 and $96 \%$, respectively).

Conclusions: quantification of microalbuminuria could correctly predict the absence of preeclampsia but was less accurate to predict the occurrence of the disease in diabetic pregnancies.

KEYWORDS: Microalbuminuria, Diabetes mellitus. Preeclampsia. Complications of pregnancy.

\section{Conflito de interesses: não relatado}

\section{Referências}

1. Koivisto V, Groop PH, Huttunen NP, et al. Diabetic nephropathy-screening, follow-up and treatment. Nephropathy study groups of the Finnish Diabetes Organization. Nord Med 1997; 112:154-62.

2. Cruz HMM, Cruz J. Microalbuminúria na hipertensão essencial. Rev Bras Hipertens 1998; 5:242-7.

3. Bar J, Hod M, Erman A, Kaplan B, Ovadia J, Schoenfeld A. Microalbuminuria in early pregnancy in normal and high-risk patients. Early Pregnancy 1996; 2:197-200.
4. Jiménez Díaz ME. Importancia clinica de la microalbuminuria en diabéticos. Rev Costarric Cienc Med 1996; 17:47-55.

5. Durruty A P. Microalbuminuria en diabeticos no insulinodependientes. Bol Hosp San Juan de Dios 1994; 41:167-72.

6. Luft FC. Microalbuminuria and essential hypertension: renal and cardiovascular implications. Curr Opin Nephrol Hypertens 1997; 6:553-7.

7. Kitzmiller JL, Brown ER, Phillippe M, et al. Diabetic nephropathy and perinatal outcome. Am J Obstet Gynecol 1981; 141:741-51.

8. Kimmerle R, Zas RP, Cupisti S, et al. Pregnancies in women with diabetic nephropathy: long-term outcome for mother and child. Diabetologia 1995; 38:227-35.

9. Winocour PH, Taylor RJ. Early alterations of renal function in insulin-dependent diabetic pregnancies and their importance in predicting pre-eclamptic toxaemia. Diabetes Res 1989; 10:159-64.

10.Combs CA, Rosenn B, Kitzmiller JL, Khoury JC, Wheeler BC, Miodovnik M. Early-pregnancy proteinuria in diabetes related to preeclampsia. Obstet Gynecol 1993; 82:802-7.

11.Giordano DM, Dubicki B, Odierna L, Vastik JF. Valor diagnóstico de la microalbuminuria y la relación calcio/ creatinina en la detección precoz de la preeclampsia. Fac Cienc Med (Cordoba) 1993; 51:15-20.

12.Baker PN, Hackett GA. The use of urinary albumincreatinine ratios and calcium-creatinine ratios as screening tests for pregnancy-induced hypertension. Obstet Gynecol 1994; 83:745-9.

13.Bertini AM, Camano L. Diabetes mellitus e gravidez. In: Prado FC, Ramos J, Valle JR, editores. Atualização Terapêutica. 20 ${ }^{a}$ ed. São Paulo: Artes Médicas; 2001. p.1005-9.

14.American Diabetes Association. Gestational diabetes mellitus. Position Statement. Diabetes Care 2002; 25 (Suppl 1):S94-S96.

15.National High Blood Pressure Education Program Working Group on High Blood Pressure in Pregnancy. Report. Am J Obstet Gynecol 2000; 183 Suppl:S1-S22.

16.Bromham DR. The increase risk of pre-eclampsia in pregnant diabetics. J Obstet Gynaecol 1983; 3:212-5.

17. Cousins L. Obstetric complications. In: Reece EA, Coustan DR, editors. Diabetes Mellitus in Pregnancy. $2^{\text {nd }}$ ed. New York: Churchill Livingstone; 1995. p.287-303.

18.Sandoval T, Jimenes G, Uribe S, Partida-Hernandes G, Gonzales S, Arreola F. Morbimotalidad perinatal em mujeres com Diabetes Mellitus y embarazo. Ginecol Obstet Mex 1995; 63:181-5.

19.Schröder W, Heyl W, Hill-Grasshoff B, Rath W. Clinical value of detecting microalbuminuria as a risk factor pregnancy-induced hypertension in insulin-treated diabetic pregnancies. Eur J Obstet Gynecol Reprod Biol 2000; 91:155-8.

20.Nakamura T, Ito M, Yoshimura T, Mabe K, Okamura $\mathrm{H}$. Usefulness of the urinary microalbumin/creatinine ratio in predicting pregnancy-induced hypertension. Int J Gynaecol Obstet 1992; 37:99-103.

Recebido em: 8/ 1/2003 Aceito com modificações em: 24/4/2003 
Laboratório: Libbs

Anúncio: Selecta 